\title{
Three-player quantum Kolkata restaurant problem under decoherence
}

\author{
M. Ramzan \\ Department of Physics Quaid-i-Azam University \\ Islamabad 45320, Pakistan
}

(Dated: June 13, 2018)

\begin{abstract}
Effect of quantum decoherence in a three-player quantum Kolkata restaurant problem is investigated using tripartite entangled qutrit states. Amplitude damping, depolarizing, phase damping, trit-phase flip and phase flip channels are considered to analyze the behaviour of players payoffs. It is seen that Alice's payoff is heavily influenced by the amplitude damping channel as compared to the depolarizing and flipping channels. However, for higher level of decoherence, Alice's payoff is strongly affected by depolarizing noise. Whereas the behaviour of phase damping channel is symmetrical around $50 \%$ decoherence. It is also seen that for maximum decoherence $(p=1)$, the influence of amplitude damping channel dominates over depolarizing and flipping channels. Whereas, phase damping channel has no effect on the Alice's payoff. Therefore, the problem becomes noiseless one at maximum decoherence in case of phase damping channel. Furthermore, the Nash equilibrium of the problem does not change under decoherence.
\end{abstract}

Keywords: Decoherence; qutrit channels; Kolkata restaurant problem.

\section{INTRODUCTION}

Game theory a branch of applied mathematics, was initially developed for use in economics by von Neumann and Morgenstern [1] and important contributions were given by John Nash [2]. It attempts to capture mathematical behavior in strategic situations, in which an individual's success of making a choice depends on the choice of the other players. It is usually used to model the behavior of biological, economical and computer systems. During last few years, a number of classical games has been converted into the realm of quantum mechanics [3-18]. Recently, Miszczak et al. [19] has studied a qubit flip game on a Heisenberg spin chain. They have shown that being 
well aware of the dimensionality of the system, a player can achieve a mean payoff equal to almost 1. More recently, Sharif et al. [20] has proposed the quantum solution to a three-player Kolkata Restaurant problem. The Kolkata Paise Restaurant (KPR) [21] is a repeated game similar to the Minority games, played between a large number of agents having no interaction among themselves. Since quantum minority games [22-25] has attracted much attention in recent years. They have also been analyzed under the influence of decoherence by Flitney and Hollenberg [26]. It is therefore, important to study the behaviour of quantum restaurant problem in the presence of environmental influences.

Since, it is not possible to completely isolate a quantum system from its environment. Therefore, it is important to study the system-environment dynamics in the presence of environmental effects. Quantum games may provide a feasible platform for implementing quantum information processing in physical systems [27] and can be used to probe the influence of decoherence in such systems [6, 28-31]. In this connection, quantum channels provide a natural theoretical framework for the study of decoherence in noisy quantum communication systems. Quantum error correction [32-33] and entanglement purifications [34] can be employed to avoid the problem of decoherence.

In this paper, the effect of quantum decoherence in a three-player quantum Kolkata restaurant problem is studied using entangled qutrit states. By considering different noisy qutrit channels parameterized by decoherence parameter $p$ such that $p \in[0,1]$. The lower and upper limits of decoherence parameter represent the fully coherent and fully decohered system, respectively. It is seen that for lower level of decoherence, amplitude damping channel heavily influences the payoffs as compared to the depolarizing and flipping channels. However, for higher level of decoherence, the payoff is strongly affected by depolarizing noise. On the other hand, the behaviour of phase damping channel is symmetrical around $50 \%$ decoherence. Furthermore, the Nash equilibrium of the problem does not change under decoherence.

\section{DECOHERENCE AND QUANTUM KOLKATA RESTAURANT PROBLEM}

In the Kolkata Paise Restaurant (KPR) problem, $N$ non-communicating agents have to choose between $n$ choices. The agents receive a gain in their utility if their choice is not too crowded, i.e. the number of agents that made the same choice is under some threshold limit. The choices can also have different values of utility associated with them, accounting for a preference profile over the set of choices. Therefore, in KPR, $N$ prospective customers choose from $N$ restaurants each evening in a parallel decision mode. Each restaurant have identical price but different rank $k$ 
(agreed by the all the $N$ agents) and can serve only one customer. If more than one agents arrive at any restaurant on any evening, one of them is randomly chosen and is served and the rest do not get dinner that evening.

For the sake of simplicity, let the three agents, Alice, Bob and Charlie have three possible choices: security 0 , security 1 and security 2 . They receive a payoff $\$=1$ if their choice is unique, otherwise they receive $\$=0$. Therefore, the game is a one shoot game, that is, it is a non-iterative, and the agents have no information from previous rounds. Since the agents cannot communicate, therefore, there is nothing left to do other than randomizing between the choices. Randomization gives the agent $i$ an expected payoff of $E^{c}(\$)=4 / 9$, where the superscript $c$ represents the classical strategy.

In this problem, let Alice, Bob and Charlie share a general tripartite entangled qutrit state of the form

$$
\rho_{i n}=f\left|\Psi_{i n}\right\rangle\left\langle\Psi_{i n}\right|+\frac{(1-f)}{27} I_{27}
$$

where the parameter $f$ controls the degree of entanglement and

$$
\left|\Psi_{i n}\right\rangle=\frac{1}{\sqrt{3}}(|000\rangle+|111\rangle+|222\rangle)
$$

In order to analyze the effect of entanglement, another general initial state is also considered as given below

$$
\left|\Psi_{i n}\right\rangle=\sin \theta \cos \phi|000\rangle+\sin \theta \sin \phi|111\rangle+\cos \theta|222\rangle
$$

where $0 \leq \theta \leq \pi$ and $0 \leq \theta \leq 2 \pi$. If we set $\theta=\pi / 4,3 \pi / 4$ and $\phi= \pm \cos ^{-1}(1 / \sqrt{3})$ in the above equation, the three-qutrit state becomes the maximally entangled state. The strategies of the players can be defined by the unitary operator $U$ acting on the initial qutrit state of the problem given as [35]

$$
U=\left[\begin{array}{ccc}
z_{1} & \bar{\omega}_{1} & \bar{z}_{2} \omega_{3}-\bar{z}_{3} \omega_{2} \\
z_{2} & \bar{\omega}_{2} & \bar{z}_{3} \omega_{1}-\bar{z}_{1} \omega_{3} \\
z_{3} & \bar{\omega}_{3} & \bar{z}_{1} \omega_{2}-\bar{z}_{2} \omega_{1}
\end{array}\right]
$$

where

$$
\vec{z}=\left[\begin{array}{c}
\sin \theta \cos \phi e^{i \alpha_{1}} \\
\sin \theta \sin \phi e^{i \alpha_{2}} \\
\cos \theta e^{i \alpha_{3}}
\end{array}\right]
$$


and

$$
\vec{\omega}=\left[\begin{array}{c}
\cos \chi \cos \theta \cos \phi e^{i\left(\beta_{1}-\alpha_{1}\right)}+\sin \chi \sin \phi e^{i\left(\beta_{2}-\alpha_{1}\right)} \\
\cos \chi \cos \theta \sin \phi e^{i\left(\beta_{1}-\alpha_{2}\right)}-\sin \chi \cos \phi e^{i\left(\beta_{2}-\alpha_{2}\right)} \\
-\cos \chi \sin \theta e^{i\left(\beta_{1}-\alpha_{3}\right)}
\end{array}\right]
$$

where $0 \leq \chi \leq \pi / 2$ and $0 \leq \beta_{1}, \beta_{2} \leq 2 \pi$. After the action of players unitary operators the state of the game transform to

$$
\rho_{\dot{f}}=\left(U_{A}^{\dagger} \otimes U_{B}^{\dagger} \otimes U_{C}^{\dagger}\right)\left(\left|\Psi_{i n}\right\rangle\left\langle\Psi_{i n}\right|\right)\left(U_{A} \otimes U_{B} \otimes U_{C}\right)
$$

The evolution of the state of a quantum system in a noisy environment can be described by the super-operator $\Phi$ in the Kraus operator representation as [1]

$$
\tilde{\rho}_{f}=\Phi \rho_{f}=\sum_{k} E_{k} \rho_{f} E_{k}^{\dagger}
$$

where the Kraus operators $E_{i}$ satisfy the following completeness relation

$$
\sum_{k} E_{k}^{\dagger} E_{k}=I
$$

We have constructed the Kraus operators for the game from the single qutrit Kraus operators (as given in equations (9-11) below) by taking their tensor product over all $n^{2}$ combination of $\pi(i)$ indices

$$
E_{k}=\underset{\pi}{\otimes} e_{\pi(i)}
$$

where $n$ is the number of Kraus operators for a single qutrit channel. The single qutrit Kraus operators for the amplitude damping channel are given by [36]

$$
E_{0}=\left(\begin{array}{ccc}
1 & 0 & 0 \\
0 & \sqrt{1-p} & 0 \\
0 & 0 & \sqrt{1-p}
\end{array}\right), E_{1}=\left(\begin{array}{ccc}
0 & \sqrt{p} & 0 \\
0 & 0 & 0 \\
0 & 0 & 0
\end{array}\right), E_{2}=\left(\begin{array}{ccc}
0 & 0 & \sqrt{p} \\
0 & 0 & 0 \\
0 & 0 & 0
\end{array}\right)
$$

Similarly, the single qutrit Kraus operators for the phase damping channel are given as [36] 


$$
E_{0}=\sqrt{1-p}\left(\begin{array}{ccc}
1 & 0 & 0 \\
0 & 1 & 0 \\
0 & 0 & 1
\end{array}\right), E_{1}=\sqrt{p}\left(\begin{array}{ccc}
1 & 0 & 0 \\
0 & \omega & 0 \\
0 & 0 & \omega^{2}
\end{array}\right)
$$

where $\omega=e^{\frac{2 \pi i}{3}}$. The single qutrit Kraus operators for the depolarizing channel are given by [37]

$$
\begin{gathered}
E_{0}=\sqrt{1-p} I, E_{1}=\sqrt{\frac{p}{8}} Y, E_{2}=\sqrt{\frac{p}{8}} Z, E_{3}=\sqrt{\frac{p}{8}} Y^{2}, E_{4}=\sqrt{\frac{p}{8}} Y Z \\
E_{5}=\sqrt{\frac{p}{8}} Y^{2} Z, E_{6}=\sqrt{\frac{p}{8}} Y Z^{2}, \quad E_{7}=\sqrt{\frac{p}{8}} Y^{2} Z^{2}, \quad E_{8}=\sqrt{\frac{p}{8}} Z^{2}
\end{gathered}
$$

where

$$
Y=\left(\begin{array}{lll}
0 & 1 & 0 \\
0 & 0 & 1 \\
1 & 0 & 0
\end{array}\right), \quad Z=\left(\begin{array}{ccc}
1 & 0 & 0 \\
0 & \omega & 0 \\
0 & 0 & \omega^{2}
\end{array}\right)
$$

The single qutrit Kraus operators for the phase flip channel are given by

$$
E_{0}=\left(\begin{array}{ccc}
1 & 0 & 0 \\
0 & \sqrt{1-p} & 0 \\
0 & 0 & \sqrt{1-p}
\end{array}\right), E_{1}=\left(\begin{array}{ccc}
0 & \sqrt{p} & 0 \\
0 & 0 & 0 \\
0 & 0 & 0
\end{array}\right), E_{2}=\left(\begin{array}{ccc}
0 & 0 & \sqrt{p} \\
0 & 0 & 0 \\
0 & 0 & 0
\end{array}\right)
$$

and the single qutrit Kraus operators for the trit-phase flip channel are given by

$$
\begin{aligned}
& E_{0}=\sqrt{1-\frac{2 p}{3}}\left(\begin{array}{lll}
1 & 0 & 0 \\
0 & 1 & 0 \\
0 & 0 & 1
\end{array}\right), E_{1}=\sqrt{\frac{p}{3}}\left(\begin{array}{ccc}
0 & 0 & e^{\frac{2 \pi i}{3}} \\
1 & 0 & 0 \\
0 & e^{\frac{-2 \pi i}{3}} & 0
\end{array}\right), \\
& E_{2}=\sqrt{\frac{p}{3}}\left(\begin{array}{ccc}
0 & e^{\frac{-2 \pi i}{3}} & 0 \\
0 & 0 & e^{\frac{2 \pi i}{3}} \\
1 & 0 & 0
\end{array}\right), E_{3}=\sqrt{\frac{p}{3}}\left(\begin{array}{ccc}
0 & e^{\frac{2 \pi i}{3}} & 0 \\
0 & 0 & e^{\frac{-2 \pi i}{3}} \\
1 & 0 & 0
\end{array}\right)
\end{aligned}
$$

where $p=1-e^{-\Gamma t}$ represents the quantum noise parameter usually termed as decoherence parameter. Here the bounds $[0,1]$ of $p$ correspond to $t=0, \infty$ respectively. The final state of the game 
after the action of the channel can be written as

$$
\rho_{f}=\Phi_{p}\left(\rho_{f}\right)
$$

where $\Phi_{\alpha}$ is the super-operator realizing the quantum channel parametrized by the real number $p$ (decoherence parameter). The payoff operator for $i^{\text {th }}$ player (say Alice) can be written as

$$
\begin{aligned}
P_{A} & =\sum_{x_{3}, x_{2}, x_{1}=0}^{2}\left|x_{3} x_{2} x_{1}\right\rangle\left\langle x_{3} x_{2} x_{1}\right|, x_{3} \neq x_{2} \neq x_{1} \\
+\sum_{x_{3}, x_{2}, x_{1}=0}^{2}\left|x_{3} x_{2} x_{1}\right\rangle\left\langle x_{3} x_{2} x_{1}\right|, x_{3} & =x_{2} \neq x_{1}
\end{aligned}
$$

The payoff of $i^{\text {th }}$ player can be calculated as

$$
E_{i}(\$)=\operatorname{Tr}\left\{P_{A} \tilde{\rho}_{f}\right\}
$$

where Tr represents the trace of the matrix. The optimal strategy for players is found to be $U_{\mathrm{opt}}$ given by

$$
U_{\text {opt }}\left(\theta, \phi, \chi, \alpha_{1}, \alpha_{2}, \alpha_{3}, \beta_{1}, \beta_{2}\right)=\left(\frac{\pi}{4}, \cos ^{-1}(1 / \sqrt{3}), \frac{\pi}{4}, \frac{5 \pi}{18}, \frac{5 \pi}{18}, \frac{5 \pi}{18}, \frac{\pi}{3}, \frac{11 \pi}{6}\right)
$$

It is seen that the results are consistent with Ref. [20] for $p=0$.

In order to interpret the effect of decoherence on the three-player quantum Kolkata restaurant problem, different graphs has been plotted as a function of decoherence parameter. In figure (1), Alice's payoff is plotted as a function of decoherence parameter $p$ for (a) $f=0.2$, (b) $f=0.5$, (c) $f=1$ and $\theta=\frac{\pi}{4}, \phi=\cos ^{-1}(1 / \sqrt{3})$ for amplitude damping, depolarizing, phase damping, trit-phase flip and phase flip channels, where AD, Dep, PD, TPF and PF represent the amplitude damping, depolarizing, phase damping, trit-phase flip and phase flip channels respectively. It is seen that Alice's payoff is heavily influenced by the amplitude damping channel as compared to the depolarizing and flipping channels. In figures (2 and 3), Alice's payoff is plotted as a function of $\theta$ and $\phi$ for $p=0.3$ and $p=0.7$ (a) amplitude damping, (b) phase damping, (c) depolarizing and (d) trit-phase flip channels, respectively, for the state of equation (3). It is seen that for higher level of decoherence (see figure 3), Alice's payoff is strongly affected by depolarizing noise. Whereas the behaviour of phase damping channel remains symmetrical around $50 \%$ decoherence. In figures (4), Alice's payoff is plotted as a function of $\theta$ and $\phi$ for $p=1$ (a) amplitude damping, (b) 
phase damping, (c) depolarizing and (d) trit-phase flip channels, respectively. It is shown that for maximum decoherence i.e. $p=1$, amplitude damping channel dominates over the depolarizing and flipping channels having considerable reduction in the payoff. Whereas, phase damping channel has no effect on the Alice's payoff. In case of phase damping channel, the problem becomes noiseless at maximum decoherence. However, maximal entanglement gives the maximum payoff (6/9 at $p=0$ ) and it reduces as one changes the degree of entanglement from its maxima or introduces the value of decoherence parameter $p>1$. Furthermore, the Nash equilibrium of the problem does not change under decoherence.

\section{CONCLUSIONS}

Quantum three-player Kolkata restaurant problem is investigated in the presence of decoherence using tripartite entangled qutrit states using amplitude damping, depolarizing, phase damping, tritphase flip and phase flip channels. It is seen that for lower level of decoherence, amplitude damping channel heavily influences the payoffs as compared to the depolarizing and flipping channels. However, for higher level of decoherence, the payoff is strongly affected by depolarizing noise. It is also seen that for maximum level of decoherence, amplitude damping channel dominates over the depolarizing and flipping channels. Whereas, phase damping channel has no effect on the Alice's payoff at $p=1$. Therefore, the problem becomes noiseless at maximum decoherence for phase damping channel only. Furthermore, the Nash equilibrium of the problem does not change under decoherence.

[1] von Neumann J and Morgenstein O, The theory of games and economic behaviour, Princeton University Press, Princeton, NJ (1944).

[2] Nash J, Equilibrium points in n-person games, Proc. National Academy of Science 36 p. 48 (1950).

[3] Meyer D A 1999 Phys. Rev. Lett. 821052

[4] Eisert J, Wilkens M and Lewenstein M 1999 Phy. Rev. Lett. 833077

[5] Marinatto L and Weber T 2000 Phys. Lett. A 272291

[6] Flitney A P and Abbott D 2005 J. Phys. A 38449

[7] Cheon T and Iqbal A 2008 J. Phys. Soc. Japan 77024801

[8] Iqbal A and Abbott D 2009 J. Phy. Soc. Japan 78014803

[9] Iqbal A, Cheon T and Abbott D 2008 Phys. Lett. A 3726564

[10] Iqbal A and Toor A H 2001 Phys. Lett. A 280249 
[11] Flitney A P and Abbott D 2002 Phys. Rev. A 65062318

[12] Iqbal A and Toor A H 2002 Phys. Rev. A 65022036

[13] Eisert J and Wilkens M 2000 J. Mod. Opt. 472543

[14] Ramzan M and Khan M K 2008 J. Phys. A: Math. Theor. 41435302

[15] Flitney A P, Ng J and Abbott D 2002 Physica A 31435

[16] Iqbal A and Toor A H 2002 Phys. Lett. A 293103

[17] Johnson N F 2001 Phys. Rev. A 63 020302(R)

[18] D'Ariano G M, Gill R D, Keyl M, Kuemmerer B, Maassen H and Werner R F 2002 Quant. Inf. Comp. 2355

[19] Miszczak J A, Gawron P and Puchala Z, (arXiv:1108.0642 [quant-ph], 2011).

[20] Puya Sharif and Hoshang Heydari, (arXiv:1111.1962 [quant-ph], 2011).

[21] Chakrabarti A S, Chakrabarti B K, Chatterjee A, Mitra M, Physica A 388 (2009) 2420-2426.

[22] Benjamin S C and Hayden P M 2001, Phys. Rev. A, 64, 030301(R).

[23] Chen Q, Wang Y, 2004 Physics Letters A, A 327, 98,102

[24] A. P. Flitney, A. Greentree, (Elsiever Science, feb 2008).

[25] C. Schmid, A.P. Flitney, (arXiv:0901.0063v1 [quant-ph], 2008).

[26] Flitney A P and Hollenberg L C L 2007 Quantum Inf. Comput. 7111

[27] Pakula I, 2007 Phys. Stat. Sol. B 244(7), 2513

[28] Ramzan M, Nawaz A, Toor A H and Khan M K 2008 J. Phys. A: Math. Theor. 41055307

[29] Gawron P and Sladkowski J 2008 Int. J. Quant. Info. 6667

[30] Chen L K, Ang H, Kiang D Kwek L C and Lo C F 2003 Phys. Lett. A 316317

[31] Ramzan M and Khan M K 2010 Quant. Inform. Process. 9, 667

[32] Steane A 1996 Phys. Rev. Lett. 77793

[33] Preskill J 1998 Proc. R. Soc. Lond. A 454385

[34] Deutsch D, Ekert A, Josza R, Macchiavello C, Popescu S and Sanpera A 1996 Phys. Rev. Lett. 772818

[35] Mathur M and Sen D 2001 J.Math.Phys. 42 4181-4196.

[36] Gregg jeager 2007 Journal of modern optics $\mathbf{5 4} 1$

[37] Berg A 2002 IEEE Transaction on Information Theory 483096 


\section{Figures captions}

Figure 1. (Color online). Alice's payoff is plotted as a function of decoherence parameter $p$ for (a) $f=0.2$, (b) $f=0.5$, (c) $f=1$ and $\theta=\frac{\pi}{4}, \phi=\cos ^{-1}(1 / \sqrt{3})$ for amplitude damping, depolarizing, phase damping, trit-phase flip and phase flip channels.

Figure 2. (Color online). Alice's payoff is plotted as a function of $\theta$ and $\phi$ for $p=0.3$ (a) amplitude damping, (b) phase damping, (c) depolarizing and (d) trit-phase flip channels for the state of equation.

Figure 3. (Color online). Alice's payoff is plotted as a function of $\theta$ and $\phi$ for $p=0.7$ (a) amplitude damping, (b) phase damping, (c) depolarizing and (d) trit-phase flip channels for the state of equation.

Figure 4. (Color online). Alice's payoff is plotted as a function of $\theta$ and $\phi$ for $p=1$ (a) amplitude damping, (b) phase damping, (c) depolarizing and (d) trit-phase flip channels for the state of equation. 

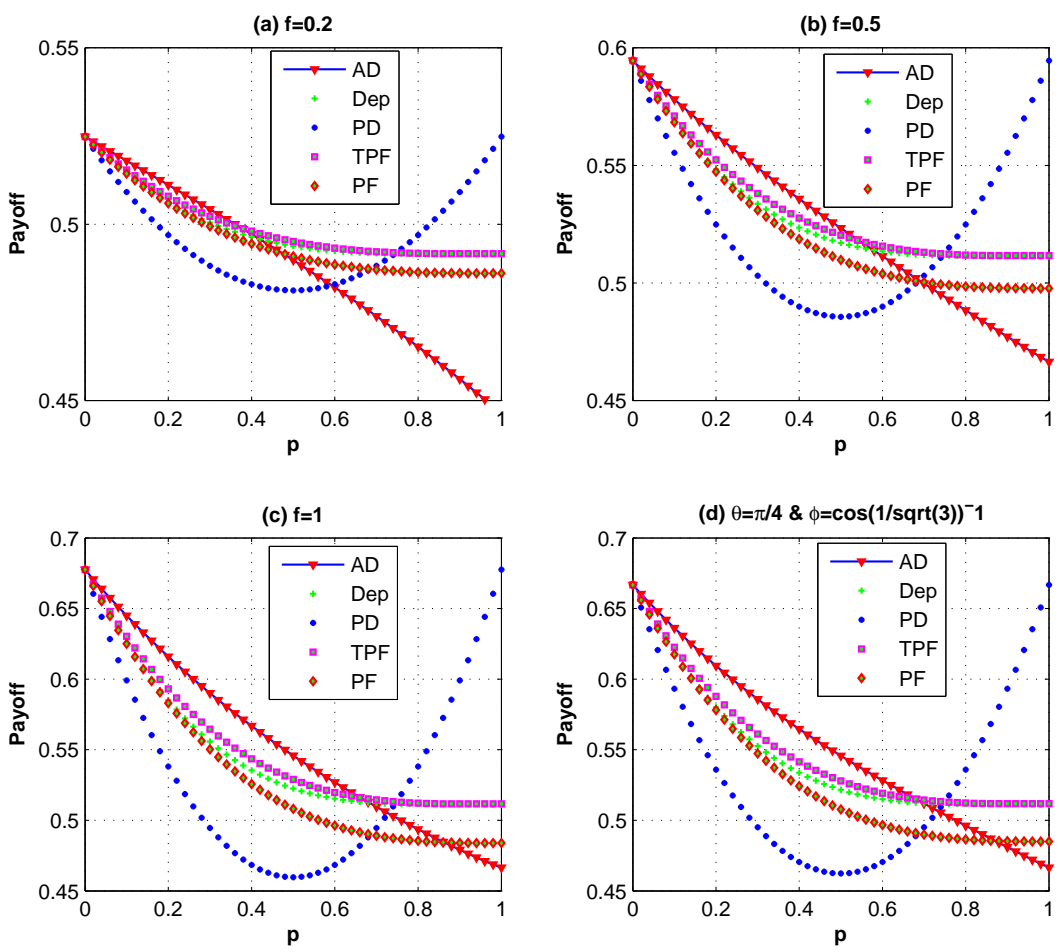

FIG. 1: (Color onlinn)

Alim's nornff in nl
(a) Amplitude Damping

(b) $f=0.5$, (c) $f=$ trit-phase flip and ]

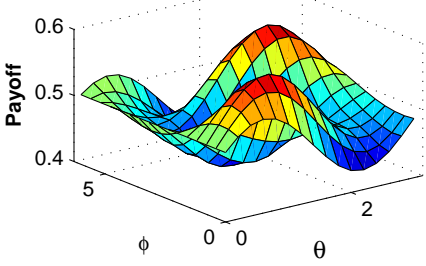

(c) Depolarizing

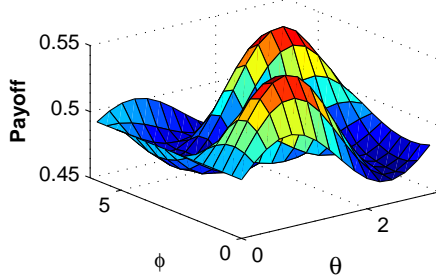

(b) Phase damping

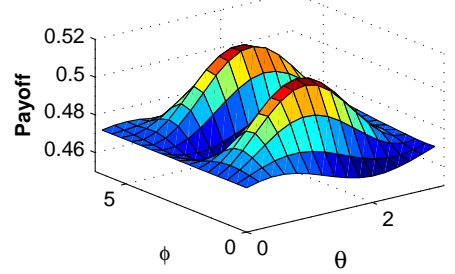

(d) Trit-phase flip

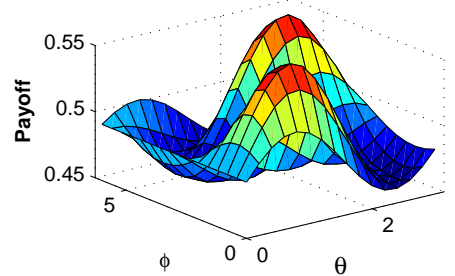

FIG. 2: (Color online). Alice's payoff is plotted as a function of $\theta$ and $\phi$ for $p=0.3$ (a) amplitude damping, (b) phase damping, (c) depolarizing and (d) trit-phase flip channels for the state of equation. 

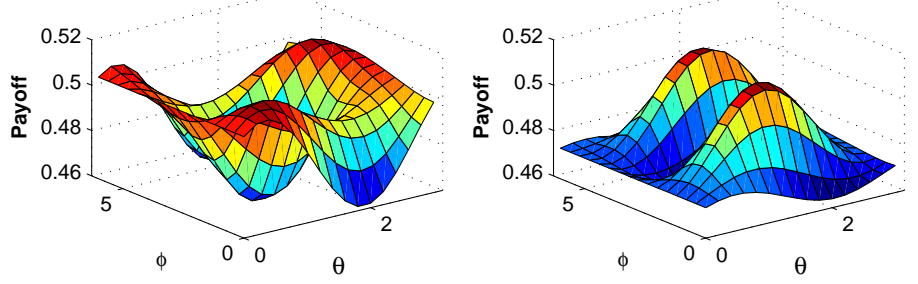

(c) Depolarizing
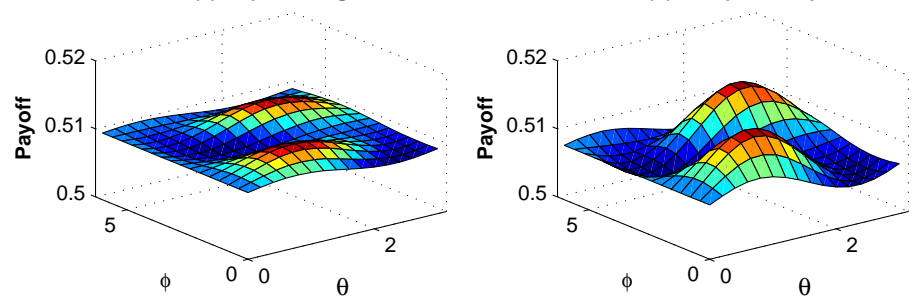

FIG. 3: (Color online). Alice's payoff is plotted as a function of $\theta$ and $\phi$ for $p=0.7$ (a) amplitude damping, (b) phase damping, (c) depolarizing and (d) trit-phase flip channels for the state of equation.
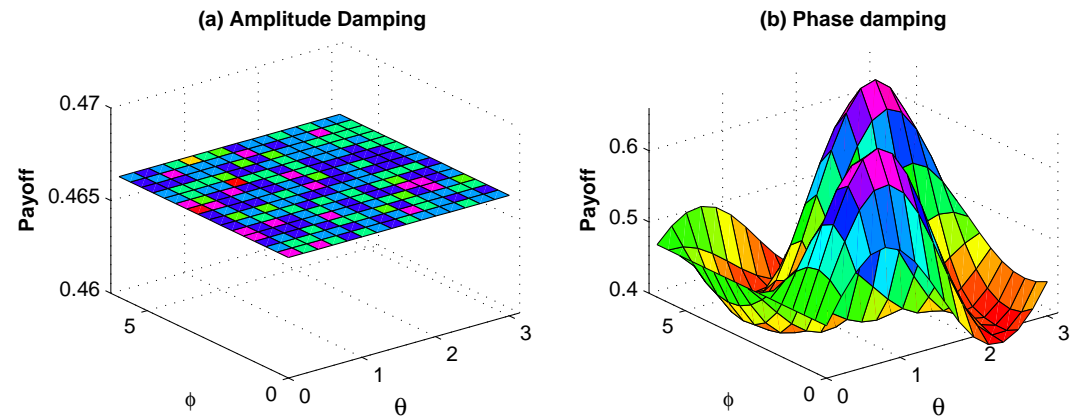

(c) Depolarizing

(d) Trit-phase flip
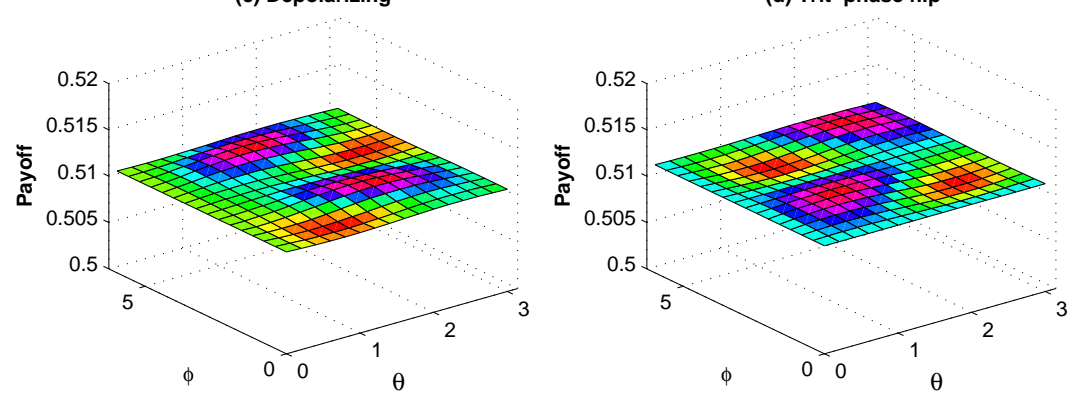

FIG. 4: (Color online). Alice's payoff is plotted as a function of $\theta$ and $\phi$ for $p=1$ (a) amplitude damping, (b) phase damping, (c) depolarizing and (d) trit-phase flip channels for the state of equation. 